\title{
A 4-strain Probiotic Supplement Influences Gut Microbiota Composition and Gut Wall Function in Patients with Ulcerative Colitis
}

Jonas Ghyselinck ${ }^{1}$, Lynn Verstrepen ${ }^{1}$, Frédéric Moens ${ }^{1}$, Pieter Van den Abbeele ${ }^{1}$, Jawal Said², Barry Smith ${ }^{3}$, Ingvar Bjarnason ${ }^{4}$, Abdul W. Basit ${ }^{5}$, Simon Gaisford, ${ }^{5, *}$

1ProDigest BV, Technologiepark 94, 9052 Ghent, Belgium

${ }^{2}$ Cernotas Ltd, Sandy Farm, The Sands, Farnham, Surrey, GU10 1PX, UK

${ }^{3}$ Symprove Ltd, Sandy Farm, The Sands, Farnham, Surrey, GU10 1PX, UK

${ }^{4}$ Department of Gastroenterology, King's College Hospital, Denmark Hill, London, SE5

9RS, UK

${ }^{5}$ UCL School of Pharmacy, University College London, 29-39 Brunswick Square, London, WC1N 1AX, UK

*Corresponding author

email: s.gaisford@ucl.ac.uk

Tel: $+44(0) 2077535868$

Fax: $+44(0) 2077535942$ 


\section{Abstract}

Symprove, a multi-strain probiotic, has been shown to exert a mild antiinflammatory effect in patients with ulcerative colitis (UC). We examined stool samples from 3 patients with UC in order to create microbiotas in an in-vitro gut model. The effects of Symprove on bacterial diversity and metabolic activity in the microbiotas was evaluated over $48 \mathrm{~h}$. In addition, the influence of probiotic dosing on epithelial tight-junction integrity, production of inflammatory markers and wound healing were evaluated in cell culture models. The relative proportions of the main bacterial phyla in UC patients differed from those of healthy subjects studied previously; levels of Firmicutes were lowered and levels of Bacteroidetes were raised. Addition of Symprove changed the bacterial composition in the microbiotas over a $48 \mathrm{~h}$ period. Several other factors generally implicated in good gut health changed after dosing with probiotic; production of short chain fatty acids (SCFAs) and lactate was stimulated, levels of anti-inflammatory cytokines (IL-6, IL-10) increased, levels of pro-inflammatory cytokines and chemokines (MCP-1 and IL-8) decreased, epithelial tight junction integrity improved and wound healing occurred faster than a control. The results imply it is not the simple addition of probiotic bacteria that improves gut health. Rather, the probiotic bacteria generate lactate, which then stimulates growth of commensal gut bacteria, raising SCFA levels (particularly butyrate). The increased butyrate concentration positively influences inflammation response and time of wound healing.

\section{Key words:}

Probiotic; short-chain fatty acid; lactic acid bacteria; inflammatory bowel disease; ulcerative colitis 


\section{Introduction}

Irritable bowel syndrome (IBS) and inflammatory bowel disease (IBD) are two of the most common gastrointestinal diseases and are frequently associated with similar symptoms. IBS is mostly a non-inflammatory condition while IBD is associated with significant intestinal inflammation proportional to the symptoms. The two most prevalent disease states of IBD are Crohn's disease (CD) and ulcerative colitis (UC). In no case is the precise cause of the illness known. With the introduction of molecular microbiotic techniques there has been renewed interest in the possibility that the microbiome may play a significant pathophysiological role in these diseases.

The human intestinal microbiota is thought to be critically important to proper gut function and maintenance of health (Guarner, 2006). Study of the microbiome is complex and compounded by age, gender, diet, ethnicity, method of birth and numerous other environmental factors. Nevertheless, it appears that, in common with many intestinal and extra-intestinal conditions, patients with IBD have an overall change in composition of their gut bacteria compared with healthy subjects; i.e. have dysbiosis. The bacteria that make up the microbiome interact with each other as well as with the human host in a symbiotic way in healthy people, but it is increasingly evident that when the microbiome is altered, as it is in many conditions, that this may be associated with disease and exacerbation of disease. The way this is brought about in patients with IBD is somewhat speculative. Because the microbiota collectively produces a wide range of compounds (such as short-chain fatty acids, SCFA, vitamins and signalling molecules) some of these may invoke an inflammatory 
response from the gut which may underpin the development and/or progression of IBD.

Dysbiosis is uniformly demonstrated in IBD patients (for instance, Machiels et al, 2014; Manichanh et al, 2006; Sokol et al, 2008; Walker et al, 2011) but the changes in bacterial phyla mostly show a reduction in the levels of Firmicutes and an elevation in levels of Bacteroidetes, rather than specific pathognomonic changes (Yan and Li, 2020, Halfvarson et al, 2017);

Treatment of active IBD is variably staged; first line treatment involves the use of 5aminosalicylic acids, antibiotics and corticosteroids (Varum et al, 2020a, 2020b). Azathioprine, an immunomodulator and related agents represent second line agents and a variety of biologicals the third stage (Yadav et al, 2016). Many of these agents may be detrimental to the gut microbiota (Vangoitsenhoven and Cresci, 2020). Accordingly, it is suggested that probiotic supplementation, as an adjunct to conventional therapy, may be beneficial (Ud Din et al, 2020) and certain probiotics have been shown to improve symptoms and/or reduce intestinal inflammation in UC in randomised controlled trials in man (Bjarnason et al, 2019).

We have shown that dosing with a live probiotic suspension (Symprove ${ }^{\mathrm{TM}}$, an aqueous probiotic suspension containing Lactobacillus acidophilus NCIMB 30175, Lactobacillus plantarum NCIMB 30173, Lactobacillus rhamnosus NCIMB 30174 and Enterococcus faecium NCIMB 30176) changed the proportions of species in the human gut microbiota in an in-vitro model (Moens et al, 2019). Symprove showed a 
high degree of gastric acid tolerance during in-vitro testing (Fredua-Agyeman and Gaisford, 2015) and it has undergone rigorous efficacy studies in a number of intestinal diseases including IBS (Sisson et al, 2014), diverticular disease (Kvasnovsky et al, 2017) and UC (Bjarnason et al, 2019). Symprove has been shown to supress Clostridium difficile (Fredua-Agyeman et al, 2017), Escherichia coli, methicillinresistant Staphylococcus aureus (MRSA) and Shigella sonnei (Dodoo et al, 2019) invitro.

Clinical acceptance and use of probiotic supplements in the management of IBD will only occur if a clinically significant effect is demonstrated in appropriate randomised, placebo-controlled trials. However, it is important to study if such outcomes are associated with the probiotic bacteria integrating and assimilating into an existing gut microbiota and/or causing a change in the proportion of species it contains and their associated metabolic activities.

We aimed here to determine whether Symprove altered bacterial proportions in the microbiotas of patients with IBD (specifically UC). The experimental difficulties of making such measurements in-vivo meant we employed an in-vitro dynamic, multicompartment gastrointestinal model (the simulator of the human intestinal microbial ecosystem, equipped with a mucosal compartment, $\left.\mathrm{M}-\mathrm{SHIME}{ }^{\circledast}\right)$, populated with microbiotas from three patients with diagnosed UC (the ability to conduct the investigation with microbiotas from diseased patients is a key advantage of this technique). Full details of the experimental arrangement of the M-SHIME ${ }^{\circledR}$ system are described elsewhere (Van den Abbeele et al, 2010, 2012, 2013). An in-vitro cell 
culture model was used to determine epithelial tight junction integrity, inflammatory response and time of wound healing.

\section{Materials and Methods}

2.1 M-SHIME ${ }^{\circledR}$ testing: Symprove was obtained from Symprove Ltd and used as received. Experiments were performed using the $\mathrm{M}-\mathrm{SHIME}{ }^{\circledR}$ system (Van den Abbeele et al, 2010, 2012, 2013) configured for short-term batch experiments. This involved fermentation of faecal samples (from three human donors, see below) in single vessels in the presence or absence of the probiotic bacteria in Symprove. A sugar-depleted nutritional medium $(56 \mathrm{~mL}$ ) buffered at $\mathrm{pH} 6.5$, containing $5.9 \mathrm{~g} / \mathrm{L}$ $\mathrm{K}_{2} \mathrm{HPO}_{4}, 18.3 \mathrm{~g} / \mathrm{L} \mathrm{KH}_{2} \mathrm{PO}_{4}, 2.3 \mathrm{~g} / \mathrm{L} \mathrm{NaHCO} 3,2.3 \mathrm{~g} / \mathrm{L}$ yeast extract, $2.3 \mathrm{~g} / \mathrm{L}$ peptone, 0.6 $\mathrm{g} / \mathrm{L}$ cysteine- $\mathrm{HCl}$ and $2.3 \mathrm{~mL} / \mathrm{L}$ Tween 80 was co-administered with Symprove $(7 \mathrm{~mL})$ at the start of fermentation. A corresponding series of blank experiments were conducted by adding the basal nutritional medium to distilled water $(7 \mathrm{~mL}$, instead of Symprove). Comparison of the blank data with the Symprove data allowed the effects of the probiotic formulation to be determined.

A 7.5\% (w/v) faecal suspension was prepared from each UC donor in anaerobic phosphate buffer $\left(\mathrm{K}_{2} \mathrm{HPO}_{4} 8.8 \mathrm{~g} / \mathrm{L} ; \mathrm{KH}_{2} \mathrm{PO}_{4} 6.8 \mathrm{~g} / \mathrm{L}\right.$; sodium thioglycolate $0.1 \mathrm{~g} / \mathrm{L}$; sodium dithionite $0.015 \mathrm{~g} / \mathrm{L})$ and was inoculated $(7 \mathrm{~mL})$ into the reactors, bringing the total volume to $70 \mathrm{~mL}$. Finally, five mucin-covered microcosms were added to all colonic vessels, enabling maintenance of not only a luminal microbiota but also specific mucus-associated bacteria in the colonic regions. Each incubation was 
performed in triplicate, resulting in 18 independent incubations. Incubations were performed under anaerobic conditions for $48 \mathrm{~h}$ at $37^{\circ} \mathrm{C}$ under continuous shaking.

\subsection{Measurement of microbial metabolites and protein metabolism: SCFA levels}

(acetate, propionate and butyrate) and branched-chain fatty acids (bCFA, isobutyrate, isovalerate and isocaproate) were quantified with gas chromatography (GC) coupled to flame ionization detection (FID). After addition of 2-methyl hexanoic acid as an internal standard, $2.0 \mathrm{~mL}$ of sample was extracted with diethyl ether. The extracts were analyzed using a GC-2014 gas chromatograph (Shimadzu, Hertogenbosch, Netherlands), equipped with a GC SGE capillary column ( $30 \mathrm{~mm} x$ $0.32 \mathrm{~mm}$ ID-BP $21 \times 0.25 \mu \mathrm{m}$, Achrom, Machelen, Belgium), a flame ionization detector and a split injector. The injection volume was $1 \mu \mathrm{L}$ and the column temperature profile was set from 110 to $160^{\circ} \mathrm{C}$, with a temperature increase of $6{ }^{\circ} \mathrm{C}$ $\min ^{-1}$. The carrier gas was nitrogen and the temperature of the injector and detector were both $200^{\circ} \mathrm{C}$.

Lactate quantification was performed using a commercially available enzymatic assay kit (R-Biopharm,Darmstadt, Germany) according to the manufacturer's instructions. Ammonium analysis was performed as previously described by Van de Wiele et al (2004) by steam distillation. The ammonium in the distillate was determined titrimetrically with $\mathrm{HCl}$.

2.3 Microbial community analysis: The microbiota profiling of each colon compartment was established with 16S-targeted sequencing analysis at the 
beginning and end ( $48 \mathrm{~h}$ ) of fermentation (Ilumina). Being polymerase-chain reaction (PCR) based, microbial gene sequences are amplified until saturation is reached, meaning results at the phylogenetic level (phylum, family, genus and operational taxonomic unit, OTU) are expressed as proportional values. The 16S rRNA gene V3V4 hypervariable regions were amplified by PCR using primers 341F (5'-CCT ACG GGN GGC WGC AG -3') and 785Rmod (5'-GAC TAC HVG GGT ATC TAA KCC-3'), with the reverse primer being adapted from Klindworth et al (2013) to increase coverage. Quality control PCR was conducted using Taq DNA Polymerase with the Fermentas PCR Kit according to the manufacturers' instructions (Thermo Fisher Scientific, Waltham, MA, USA). The obtained PCR product was run along the DNA extract on a $2 \%$ agarose gel for $30 \mathrm{~min}$ at $100 \mathrm{~V}$. An aliquot of the original genomic DNA extract $(10 \mu \mathrm{L})$ was sent out to LGC genomics $\mathrm{GmbH}$ (Germany) for library preparation and sequencing on an Illumina Miseq platform with $v 3$ chemistry with the primers mentioned above.

2.4. Caco-2/THP1-blue ${ }^{T M}$ co-culture model: The co-culture experiment was performed as previously described (Daguet et al, 2016). Caco-2 cells (HTB-37; American Type Culture Collection) were seeded in 24-well semi-permeable inserts ( $0.4 \mu \mathrm{m}$ pore size) at a density of $1 \times 10^{5}$ cells/insert. Caco- 2 monolayers were cultured for 14 days, with three changes of medium per week, until a functional monolayer with a transepithelial electrical resistance (TEER) of more than $300 \Omega \mathrm{cm}^{2}$ was obtained (measured with a Millicell ERS-2 epithelial volt-ohm meter, Millipore). Cells were maintained in Dulbecco's Modified Eagle Medium (DMEM) containing 
glucose $(25 \mathrm{mM})$ and glutamine $(4 \mathrm{mM})$, supplemented with HEPES (10 mM) and heat-inactivated foetal bovine serum (HI-FBS, $20 \% \mathrm{v} / \mathrm{v})$.

THP1-Blue ${ }^{\text {TM }}$ cells (InvivoGen) were seeded in 24-well plates at a density of $5 \times 10^{5}$ cells/well and treated with phorbol 12-myristate 13-acetate (PMA) for $48 \mathrm{~h}$ and maintained in Roswell Park Memorial Institute (RPMI) 1640 medium containing glucose $(11 \mathrm{mM})$ and glutamine $(2 \mathrm{mM})$, supplemented with HEPES (10 mM), sodium pyruvate $(1 \mathrm{mM})$ and HI-FBS $(10 \% \mathrm{v} / \mathrm{v})$.

Before setting up the co-culture, the TEER of the Caco-2 monolayer was measured (the TEER of an empty insert was subtracted from all readings). Caco-2 bearing inserts were then placed on top of the PMA-differentiated THP1-blue ${ }^{T M}$ cells. The apical compartment (containing Caco- 2 cells) was filled with sterile-filtered $(0.22 \mu \mathrm{m})$ colonic SHIME media (diluted 1:5 v/v in Caco-2 complete medium). Cells were treated apically with sodium butyrate (12 mM, Sigma-Aldrich) as a positive control. The basolateral compartment (containing THP1-blue ${ }^{\mathrm{TM}}$ cells) was filled with Caco-2 complete medium. Cells were treated for $24 \mathrm{~h}$, after which the TEER was measured. The basolateral supernatant was then discarded and cells were stimulated on the basolateral side with Caco-2 complete medium containing ultrapure lipopolysaccharide (LPS, E. coli K12, InvivoGen). Cells were also stimulated at the basolateral side with LPS in combination with hydrocortisone (HC, Sigma-Aldrich) and medium without LPS as controls. After LPS stimulation $(6 \mathrm{~h})$ the basolateral supernatants were collected for cytokine measurement (human TNF- $\alpha$, IL-6, IL-8, IL10, CXCL10 and MCP-1) by Luminex ${ }^{\circledR}$ multiplex (Affymetrix-eBioscience) and for NF- 
$\kappa B$ activity. The THP1-Blue ${ }^{\mathrm{TM}}$ cells contain a stably transfected reporter construct allowing the expression of a secreted alkaline phosphatase (SEAP) under the control of an NF- $\kappa B$ inducible promoter. Hence, upon LPS stimulation, NF- $\kappa B$ is activated, leading to the secretion of SEAP in the basolateral medium. Then, SEAP activity was measured in the basolateral medium using the QUANTI-Blue ${ }^{T M}$ reagent (Invivogen). All measurements were performed in triplicate and cells were incubated at $37^{\circ} \mathrm{C}$ in a humidified atmosphere of air/ $\mathrm{CO}_{2}(95: 5 \mathrm{v} / \mathrm{v})$.

2.5. Scratch wound healing assay: T84 cells (Sigma-Aldrich) were seeded in 24-well plates and cultured for 7 days, with three changes of medium per week, until a complete, confluent, cell monolayer was formed. Cells were maintained in DMEM/Nutrient mixture F-12 Ham containing L-glutamine and HEPES supplemented with antibiotic/antimycotic (Gibco, Life Technologies) and 5\% HI-FBS. Cells were incubated at $37^{\circ} \mathrm{C}$ in a humidified atmosphere of air/ $\mathrm{CO}_{2}(95: 5 \mathrm{v} / \mathrm{v})$.

After 7 days of culture a scratch was created in the T84 cell monolayer, followed by treatment with $1 / 10$ diluted colonic suspensions in serum-free T84 culture medium. Images were captured with a Cytation 5 Cell Imaging Multi-mode Reader at the initial time point $(0 \mathrm{~h})$ and after incubation $(24 \mathrm{~h})$. Images were compared in order to quantify the migration rate of the cells by measuring the wound area using Image ${ }^{\circledR}$. Serum-free culture medium and $5 \mathrm{mM} \mathrm{NaB}$ (Sigma-Aldrich) were used as negative and positive controls respectively. All measurements were conducted in triplicate. 


\subsection{Statistical tests for the cell assays:}

Caco-2/THP1 co-culture: Colonic batch treatment samples were compared with their respective control samples using a two-way ANOVA with Sidak's multiple comparisons test. For the pure product, statistical significance for the TEER was calculated by using one-way ANOVA with Dunnett's multiple comparisons test and the different concentrations of Symprove were compared with CM. Statistically significant differences are represented by $\left({ }^{*}\right) .\left({ }^{*}\right),\left({ }^{* *}\right),\left({ }^{* *}\right)$ and $\left({ }^{* * *}\right)$ represent $p<0.05, p<0.01, p<0.001$ and $p<0.0001$, respectively. All statistics were performed using GraphPad Prism version 7.02 for Windows (GraphPad Software, San Diego, CA, USA).

Wound healing: Statistical significance between $\mathrm{CM}$ and sodium butyrate was calculated with an unpaired, two-tailed t-test with Welch's correction. To evaluate differences in wound area, colonic batch treatment samples were compared with their respective control samples using a two-way ANOVA with Sidak's multiple comparisons test. For pure product, statistical significance was calculated by using one-way ANOVA with Dunnett's multiple comparisons test against CM. Statistically significant differences are represented by $\left({ }^{*}\right) .\left({ }^{*}\right),\left({ }^{* *}\right),\left({ }^{* *}\right)$ and $\left({ }^{* * *}\right)$ represent $p<0.05, p<0.01, p<0.001$ and $p<0.0001$, respectively. All statistics were performed using GraphPad Prism version 7.02 for Windows (GraphPad Software, San Diego, CA, USA).

All samples were taken as biological replicates in the cell assays $(n=3 /$ donor) and statistics were performed on the average of the replicates. 
2.7. Donors. Faecal samples were obtained from three patients with UC, all in clinical remission and none recently on steroids). Samples were collected according to the ethical approval of the University Hospital Ghent (reference number: B670201836585).

\section{Results and discussion}

Table 1 shows the proportions of the five main phyla of the microbiotas of the UC donors prior to addition of Symprove. The predominant phylum was the Firmicutes, with Bacteroidetes and Actinobacteria accounting for the majority of the remaining bacteria. Firmicutes accounted for more than half the bacteria in all donors while Bacteroidetes were second most prevalent in donors 1 and 3. Donor 2 showed a high level of Actinobacteria, which were present at the expense of Bacteroidetes.

Tables 2 and 3 show the compositions of the gut microbiota, in terms of the five major phyla, for the three donors after the control and Symprove incubations (the data are shown graphically in Figure S1 and familial detail of operational taxonomic units (OTU) within phyla are given in Tables S1 and S2). Some significant changes in bacterial composition are apparent. Firstly, the proportion of Firmicutes was raised in the mucus of all three donors, and in the lumen for donor 1. Partly, this enrichment reflected the integration and proliferation of the probiotic species in Symprove, but there were contributions from other families, in particular Lachnospiraceae and to a lesser extent Streptococcaceae and Veillonellaceae in the mucus and Roseburia species in the lumen (OTU13 for donors 1 and 2 and OTU44 for 
donor 3). More specifically, Symprove resulted in a substantial increase in the relative abundance of Veillonellaceae in the luminal microbiota of donor 1, whereas for donors 2 and 3 the increased abundance of Firmicutes was due to a substantial increase in Lachnospiraceae in the mucus layer. Levels of Actinobacteria were raised in the luminal compartments of all donors, principally due to stimulation of Bifidobacteriaceae (although no specific OTUs were seen to increase in all donors; Bifidobacterium longum was enriched in donor 3 while Bifidobacterium pseudolongum was enriched in donor 2 for instance).

The application of Symprove also resulted in a small change in Proteobacteria numbers; a mild stimulatory effect was seen in the mucus while their relative numbers were lowered in the lumen (in all donors). Enrichment was primarily due to Enterobacteriaceae in donors 1 and 2 and Burkholderiaceae in donors 2 and 3. Lowering was due to loss of Burkholderiaceae (OTU52, Parasutterella excrementihominus) in donor 3 and Enterobacteriaceae (OTU1, E. coli) in donors 1 and 2. Other changes that did not reach statistical significance included stimulation of Verrucomicrobia (OTU6, A. muciniphila) in donor 1 and enrichment of Bacteroidaceae in the lumen of donor 2.

Patients with IBD exhibit reduced diversity of bacteria in their microbiotas compared with healthy controls. Walker et al (2011) demonstrated reduced numbers of Firmicutes and raised levels of Bacteroidetes in IBD patients and, in UC patients only, raised levels of Enterobacteriaceae. In a study of UC patients, Roseburia hominis and Faecalibacterium prausnitzii were reduced (Machiels, 2014). Broadly, these clinical 
observations are in agreement with the data reported here; in the donor samples prior to treatment, levels of Firmicutes were lowered in 2 of the donors and levels of Bacteroidetes were raised in all three donors.

The relative abundance of $F$. prausnitzii may be a particularly important factor in IBD because the species has been shown to exert a strong anti-inflammatory effect in the gut (Sokol et al, 2008) associated with reductions in pro-inflammatory cytokines, particularly NF- $\kappa B$ (Segain et al, 2000). Further, it is associated with high butyrate concentrations; butyrate is an important energy source for colonocytes and may stimulate production of regulatory anti-inflammatory T-cells (Arpaia et al, 2013). In this work, incubation with Symprove did not significantly increase the relative abundance of $F$. prausnitzii, but it did stimulate Roseburia species in the lumen.

Machiels et al (2014) reported reduced levels of the SCFA acetate, propionate and butyrate in UC patients while L-lactic acid levels in faeces was increased in patients with active disease. In a meta-analysis of 12 studies, Zhuang et al (2019) found acetate, valerate and total SCFA levels were reduced in UC patients.

Figure 1 shows the lactate and SCFA concentrations with and without incubation with Symprove. Concentrations of acetate, propionate and butyrate all increased with time. Acetate concentrations were raised after $6 \mathrm{~h}$ and continued to rise during the first $24 \mathrm{~h}$ of the test for all donors. In donor 1, acetate levels continued to rise during the $48 \mathrm{~h}$ of the test, while for donor $\mathrm{C}$ acetate was consumed during the 24 $48 \mathrm{~h}$ period, indicative of cross-feeding. Propionate and butyrate production started 
after $6 \mathrm{~h}$ and concentrations continued to rise for the remainder of the test for all donors. Lactate concentrations rose substantially after $6 \mathrm{~h}$ but was then actively consumed by the microbiota.

The changes in SCFA and lactate levels associated with incubation with Symprove are in keeping with previous studies (Moens et al, 2019). Lactate increases in concentration first, because of carbohydrate fermentation by the lactic-acid bacteria in Symprove. Lactate itself does not accumulate in the system, however, because it is a substrate for propionate-producing species, such as Veillonella and Megasphaera (Reichardt et al, 2014), and butyrate-producing species, such as Anaerostipes caccae and E. hallii (Duncan et al, 2004a; Louis et al, 2014; Flint et al,2015). The obtained data for the specific stimulation of certain SCFAs in the different donors correlated well with the stimulatory effect of Symprove on certain bacterial families in the different donors (see above). Whereas dosing of Symprove resulted in a general increase in SCFA production for each donor, acetate and propionate production was mainly stimulated in donor 1, whereas the highest stimulation observed for donors 2 and 3 was for butyrate. Indeed, compositional analyses revealed that Symprove mainly stimulated the growth of Veillonellaceae in the lumen of donor 1, whereas in donors 2 and 3 Symprove resulted in a significant stimulation of the Lachnospiraceae on the mucus. Members of the Veillonellaceae family will consume lactate resulting in the production of propionate and acetate (Ng and Hamilton, 1971) whereas the Lachnospiraceae contain lactate-consuming, butyrate-producing bacterial species (Duncan et al., 2004a). 
The induction of lactate cross-feeding is a key mechanism in the action of lactic acid containing probiotics, assuming that such probiotics survive the passage through the stomach and arrive in a viable state in the colon. At the same time, the increase in lactic acid concentration reduces local $\mathrm{pH}$, making the environmental conditions tougher for certain opportunistic pathogenic species. The net result is a change in the relative abundances of species in the microbiota, in favour of the beneficial commensal groups. Importantly, this is a general effect of probiotics suggesting that probiotic supplements have a potential to exert a beneficial effect in various conditions.

Acetate is produced as a by-product of saccharolytic fermentation by numerous bacterial groups (including Bifidobacteria, De Vuyst et al, 2014, Bacteroidetes,' Macy et al, 1978; Baxter et al, 2019, and acetogenic bacteria, Ragsdale and Pierce 2008) and in human faeces it accounts for more than $50 \%$ of the total SCFA, Louis et al, 2007. This accounts for the elevation in acetate levels seen in the test. Acetate is itself a substrate for many butyrate-producing species (such as F. prausnitzii and Roseburia spp' Duncan et al, 2004b) and is an essential co-substrate for butyrate synthesis from lactate or carbohydrate (Duncan et al, 2002) and so its presence stimulates the production of butyrate at the expense of lactate.

Gut microbes switch to protein metabolism, once available dietary carbohydrate has been consumed, resulting in the production of various metabolic by-products including ammonium, numerous branched-chain fatty acids, principally isobutyrate, isovalerate and isocaproate, and several amines, phenols/indoles and sulphides. Low 
levels of these compounds are desirable because they are generally viewed as detrimental to human health (Scott et al, 2013). Figure 2 shows the bCFA and ammonium concentrations recorded in the microbiotas of IBD donors following dosing with Symprove; the concentrations of bCFAs were not significantly altered, but the ammonium concentrations were lowered in all cases, relative to the control samples.

While it is difficult to state with certainty that there are distinctive and characteristic changes in gut microbiota diversity in patients with IBD, it does seem that in general levels of Firmicutes are reduced, levels of Bacteroidetes are raised and production of SCFAs is suppressed. Given that incubation with Symprove in the current study improved all three of these, it seems reasonable to suggest that this may be one of the mechanisms that mediate the anti-inflammatory effect of Symprove seen in patients with UC (Bjarnason et al, 2019).

Intestinal intercellular tight junction integrity is disrupted in UC (Artis, 2008) often because of dysbiosis of the gut microbiota. Tight-junction integrity was quantified here with an in-vitro bilayer cell model comprising epithelial-like cells (Caco-2 cells) and immune cells of human origin (THP1 cells). Table 3 shows the TEER values for Caco-2/THP1 cells treated with colonic media with and without dosing with Symprove for $24 \mathrm{~h}$. For all donors the TEER values of the controls were the same as those of the experimental controls, while following dosing with the probiotic TEER values were higher (although they did not reach statistical significance), indicating a mild protective effect on inflammation-induced tight junction integrity. Many studies 
have shown that Lactobacillus and/or butyrate have a positive influence on TEER and wound healing (for instance, Ma et al, 2012; Gudadappanavar et al, 2017). The issue is to know whether the effects arise from addition of the probiotic or stimulation of the microbiome.

We have reported previously the effect of incubating the apical side of the cell model with various concentrations of probiotic bacteria for $24 \mathrm{~h}$ (Ghyselinck et al, 2020). No increase in TEER was seen at bacterial concentrations of $10^{5}, 10^{6}$ or $10^{7}$ $\mathrm{cfu} / \mathrm{mL}$, confirming that the simple presence of the probiotic bacteria had no influence of tight-junction integrity. We also showed that exposure to sodium butyrate $(12 \mathrm{mM}$ ) for $24 \mathrm{~h}$ caused an increase in TEER to $110.3 \pm 1.3$ (Ghyselinck et al, 2020). Thus, it appears that simple addition of probiotics per se to the gut does not result in improved epithelial tight-junction integrity. Rather, gut wall barrier function improves from the increased SCFA concentration (butyrate in particular) produced by stimulation of the commensal gut bacteria. It is critical to note, though, that in order to exert an effect in-vivo, it is critical that the bacteria in a probiotic supplement survive the challenges presented by oral delivery; low stomach $\mathrm{pH}$ and high bile salt concentrations/ionic strength in the small intestine. We have shown previously that Symprove showed a particularly high degree of tolerance to acid during in-vitro testing (Fredua-Agyeman and Gaisford, 2015) and in previous MSHIME tests $99.7 \%$ of the bacteria added to the system arrived in the colonic chambers in a viable state (Moens et al, 2019). 
The inflammatory response of the cells was evaluated by measuring production of anti-inflammatory cytokines (IL-6, IL-10) and inflammatory cytokines and chemokines (TNF $\alpha$, MCP-1, CXCL 10 and IL-8). Figure 3 shows the NF- $\kappa B$ activity and Figure 4 shows the concentrations of IL- 6 and IL-10 following exposure of the cells to colonic media with and without addition of Symprove for $24 \mathrm{~h}$. For all donors, Symprove increased NF- $\mathrm{KB}$ activity and concomitant secretion of the antiinflammatory cytokines compared with the untreated controls. Upon treatment, the secretion of IL-6 and IL-10 were higher in donors 2 and 3 compared with donor 1 , in accordance with the higher butyrate production observed in these donors. Whereas the anti-inflammatory role of IL-10 is clear, the anti-inflammatory properties of IL-6 are dependent on the pathophysiological context. Indeed, IL-6 has been shown to exert positive effects on the regeneration of the intestinal epithelium and wound healing (Dann et al, 2008). Moreover, IL-6 inhibits production of pro-inflammatory cytokines (TNF $\alpha$ and IL-1 $\beta$ ) in monocytes/macrophages (Aderka et al, 1989). Figure 5 shows the concentrations of TNF $\alpha$, MCP-1, CXCL 10 and IL-8 following exposure of the cells to colonic media with and without addition of Symprove for $24 \mathrm{~h}$. Here, concentrations were highly dependent on the donor. TNF $\alpha$ secretion was decreased for all donors, but these changes were not statistically significant. For all donors, CXCL 10 secretion increased, reaching significance for donor 3 while those of MCP-1 and IL-8 decreased. Thus, overall there was a positive influence of Symprove on most markers of inflammation.

Wound healing was assessed using a scratch wound assay. In this assay, a scratch is made in the T84 cell monolayer and the closing of the resulting wound is followed 
visually. By exposing the wound to colonic media, the influence of fermentationderived metabolites on the rate and extent of wound healing can be quantified. Figure 6 shows images of the wound as created and after 24h exposure to complete medium (CM) or sodium butyrate (positive control). After $24 \mathrm{~h}$, the CM sample wound had reduced to $75 \%$ of its initial area while the positive control wound reduced to $45 \%$ of its initial area, indicating sodium butyrate acts positively to encourage wound healing.

Figure 6 also shows the wound treated with colonic media (with and without Symprove) from donor 1 (percentages of wound healing for all donors are shown in Table 4). In all cases, treatment with Symprove increased wound healing compared with the control and correlated with the increased butyrate levels observed in each donor. Again, the results suggest that butyrate is the key factor that encourages wound closure and the positive results from dosing with probiotic arise from increased SCFA concentrations from stimulation of the commensal gut bacteria.

\section{Summary}

The effects of dosing the gut microbiotas of three patients with IBD with an oral probiotic suspension were assessed. Levels of Firmicutes were lowered in 2 donors and levels of Bacteroidetes were raised in all three donors, relative to data we recorded previously for healthy individuals. Dosing with probiotic changed bacterial diversity in the microbiotas over a $48 \mathrm{~h}$ period, suggesting that probiotic supplementation may be a viable option to help rebalance microbiotas in-vivo. Several other indicators of gut health improved when dosing with the probiotic; 
production of short chain fatty acids (SCFAs) and lactate was stimulated, levels of anti-inflammatory cytokines (IL-6, IL-10) increased and levels of pro-inflammatory cytokines and chemokines (TNF $\alpha$, MCP-1 and IL-8) decreased. Tight junction integrity was improved in the presence of sterilized colonic fluid obtain after incubation with Symprove. The work suggests that supplementation of the diet of patients with IBD with a properly formulated probiotic may be a useful adjunct to standard treatment in clinic. Three principal mechanisms are proposed; (i) redressing any dysbiosis of the microbiota by increasing levels of Firmicutes and lowering levels of Bacteroidetes, (ii) increasing SCFA production (particularly butyrate) and (iii) reducing the effects of inflammation on epithelial tight junction integrity. The small number of donors means results should be interpreted with caution and need replication, but combined with human and animal data they provide a compelling indication that probiotics may be a potential and cost-effective intervention in managing patients with UC.

\section{Acknowledgements}

Symprove Ltd funded the M-SHIME studies conducted by ProDigest BV. The research team thanks the UC patient donors and Laura McCoubrey for proof reading the manuscript. 


\section{References}

Aderka, D., Le, J.M., Vilcek, J. IL-6 inhibits lipopolysaccharide-induced tumor necrosis factor production in cultured human monocytes, U937 cells, and in mice. J. Immunol. 143, 3517-3523 (1989).

Arpaia, N., Campbell, C., Fan, X., Dikiy, S., van der Veeken, J., deRoos, P., Liu, H., Cross, J.R., Pfeffer, K., Coffer, P.J., Rudensky, A.Y. Metabolites produced by commensal bacteria promote peripheral regulatory T-cell generation. Nature, 504, 451-456 (2013).

Artis D. Epithelial-cell recognition of commensal bacteria and maintenance of immune homeostasis in the gut. Nat. Rev. Immun. 8, 411-420 (2008). Baxter NT, et al. Dynamics of human gut microbiota and short-chain fatty acids in response to dietary interventions with three fermentable fibres. mBio, 10, e0256618 (2019).

Bjarnason, I., Sisson, G., Hayee, B. A randomised, double-blind, placebo-controlled trial of a multi-strain probiotic in patients with asymptomatic ulcerative colitis and Crohn's disease. Inflammopharmacology 27, 645-473 (2019).

Daguet, D., Pinheiro, I., Verhelst, A., Possemiers, S. \& Marzorati M. Arabinogalactan and fructooligosaccharides improve the gut barrier function in distinct areas of the colon in the Simulator if the Human Intestinal Microbial Ecosystem. J. Func. Foods 20, 369-379 (2016).

Dann, S.M., Spehlmann, M.E., Hammond, D.C., limura, M., Hase, K., Choi, L.J., Hanson, E., Eckmann, L. IL-6 dependent mucosal protection prevents establishment of a microbial niche for attaching/effacing lesion-forming enteric bacterial pathogens. J Immunol. 180, 6816-6926 (2018). 
De Vuyst, L., Moens, F., Selak, M. \& Leroy, F. Summer meeting 2013: growth and physiology of bifidobacteria. J. Appl. Microbiol. 116, 477-491 (2014).

De Weirdt, R., et al. Human fecal microbiota display variable patterns of glycerol metabolism. FEMS Microbiol. Ecol. 74, 601-611 (2010).

Dodoo, C.C., et al. Use of a water-based probiotic to treat common gut pathogens. Int. J. Pharm. 556, 136-141 (2019).

Duncan, S.H., Louis, P. \& Flint, H.J. Lactate-utilizing bacteria, isolated from human feces, that produce butyrate as a major fermentation product. Appl. Environ.

Microbiol. 70, 5810-5817 (2004a).

Duncan, S.H., et al. Contribution of acetate to butyrate formation by human faecal bacteria. Br. J. Nutr. 91, 915-923 (2004b).

Duncan, S.H., Barcenilla, A., Stewart, C.S., Pryde, S.E. \& Flint, H.J. Acetate utilization and butyryl coenzyme A (COA):acetate-CoA transferase in butyrate-producing bacteria from the human large intestine. Appl. Environ. Microbiol. 68, 5186-5190 (2002).

Flint, H.J., Duncan, S.H., Scott, K.P. \& Louis, P. Links between diet, gut microbiota composition and gut metabolism. Proc. Nutr. Sci. 74, 13-22 (2015).

Fredua-Agyeman, M. \& Gaisford, S. Comparative survival of commercial probiotic formulations: tests in biorelevant gastric fluids and real-time measurements using microcalorimetry. Beneficial Microbes 6, 141-151 (2015).

Fredua-Agyeman, M., et al. In vitro inhibition of Clostridium difficile by commercial probiotics: A microcalorimetric study. Int. J. Pharm. 517, 96-103 (2017). Guarner, F. Enteric gut flora in health and disease. Digestion 73(S1), 5-12 (2006) 
Gudadappanavar, A.M., Hombal, P.R., Timashetti, S.S., Javali, S.B. Influence of Lactobacillus acidophilus and Lactobacillus plantarum on wound healing in male Wistar rats - an experimental study. Int. J. Appl. Basic Med. Res. 7, 233-238 (2017).

Ghyselinck, J., et al. Influence of probiotic bacteria on gut microbiota composition and gut wall function in patients with Parkinson's Disease. Nature Parkinson's Disease, submitted.

Halfvarson, J., Brislawn, C.J., Lamendella, R., Vázquez-Baeza, Y., Walters, W.A. et al. Dynamics of the human gut microbiome in inflammatory bowel disease. Nat.

Microbiol. 2, 17004 (2017).

Klindworth, A., et al. Evaluation of general 16S ribosomal RNA gene PCR primers for classical and next generation sequencing-based diversity studies. Nucleic Acids Res. 41, e1 (2013).

Kvasnovsky, C.L., et al. A randomized double-blind placebo-controlled trial of a multistrain probiotic in treatment of symptomatic uncomplicated diverticular disease. Inflammopharmacol. 25, 499-509 (2017).

Louis, P., Scott, K.P. \& Duncan, S.H. Understanding the effects of diet on bacterial metabolism in the large intestine. J. Appl. Microbiol. 102, 1197-1208 (2007). Louis, P., Hold, G.L. \& Flint, H. The gut microbiota, bacterial metabolites and colorectal cancer. Nat. Rev. Microbiol. 12, 661-672 (2014).

Machiels, K., Joossens, M., Sabino, J., De Preter, V., Arijs, I., Eeckhaut, V., Ballet, V., Claes, K., Van Immerseel, F., Verbeke, K., Ferrante, M., Verhaegen, J., Rutgeerts, P., Vermeire, S. A decrease of the butyrate-producing species Roseburia hominis and Faecalibacterium prausnitzii defines dysbiosis in patients with ulcerative colitis. Gut 63, 1275-1283 (2014). 
$\mathrm{Ma}, \mathrm{X}$. et al. Butyrate promotes the recovering of intestinal wound healing through its positive effect on the tight junctions. J. Anim. Sci. 90, 266-268 (2012). Macy, J.M., Ljungdahl, L.G. \& Gottschalk, G. Pathway of succinate and propionate formation in Bacteroides fragilis. J. Bacteriol. 134, 84-91 (1978). Manichanh, C., Rigottier-Gois, L., Bonnaud, E., Gloux, K., Pelletier, E., Frangeul, L., Nalin, R., Jarrin, C., Chardon, P., Marteau, P., Roca, J., Dore, J. Reduced diversity of faecal microbiota in Crohn's disease revealed by a metagenomic approach. Gut, 55, 205-211 (2006).

Moens, F., et al. A four-strain probiotic exerts positive immunomodulatory effects by enhancing colonic butyrate production in vitro. Int. J. Pharm., 555, 1-10 (2019). Ragsdale, S.W. \& Pierce, E. Acetogenesis and the Wood-Ljungdahl pathway of $\mathrm{CO}_{2}$ fixation. Biochim. Biophys. Acta 1784, 1873-1898 (2008).

Ng, S.K.C., Hamilton, I.R. Lactate metabolism by Veillonella parvula. J. Bacteriol. 105, 999-1005 (1971).

Reichardt, N., et al. Phylogenetic distribution of three pathways for propionate production within the human gut microbiota. ISME J. 8, 1323-1335 (2014). Scott, K.P., Gratz, S.W., Sheridan, P.O., Flint, H.J. \& Duncan, S.H. The influence of diet on the gut microbiota. Pharmacol. Res. 69, 52-60 (2013).

Segain, J.P., Raingeard de la Blétière, D., Bourreille, A., Leray, V., Gervois, N., Rosales, C., Ferrier, L., Bonnet, C., Blottière, H.M., Galmiche, J.P. Butyrate inhibits inflammatory responses through NFkappaB inhibition: Implications for Crohn's disease. Gut, 47, 397-403 (2000). 
Sisson, G., et al. Randomised clinical trial: A liquid multi-strain probiotic vs. placebo in the irritable bowel syndrome - a 12 week double-blind study. Aliment. Pharmacol. Ther. 40, 51-62 (2014).

Sokol, H., Pigneur, B., Watterlot, L., Lakhdari, O., Bermúdez-Humarán, L.G. et al. Faecalibacterium Prausnitzii is an anti-inflammatory commensal bacterium identified by gut microbiota analysis of Crohn disease patients. Proc. Nat. Acad. Sci. USA, 105, 16731-16736 (2008).

Ng, S.K.C., Hamilton, I.R. Lactate metabolism by Veillonella parvula. J. Bacteriol. 105, 999-1005 (1971).

Ud Din, A., Hassan, A., Zhu, Y., Zhang, K., Wang, Y., Li, T., Wang, Y., Wang, G. Inhibitory effect of Bifidobacterium bifidum ATCC 29521 on colitis and its mechanism. J. Nutr. Biochem. 79, 108353 (2020).

Van de Wiele, T., Boon, N., Possemiers, S., Jacobs, H., Verstraete, W. Prebiotic effects of chicory inulin in the simulator of the human intestinal microbial ecosystem. FEMS Microbiol. Ecol. 51, 143-153 (2004).

Van den Abbeele, P., et al. Microbial community development in a dynamic gut model is reproducible, colon region specific, and selective for Bacteroidetes and Clostridium Cluster IX. Appl. Environ. Microbiol. 76, 5237-5246 (2010).

Van den Abbeele, P., et al. Incorporating a mucosal environment in a dynamic gut model results in a more representative colonization by lactobacilli. Microb.

Biotechnol. 5, 106-115 (2012).

Van den Abbeele, P., et al. Butyrate-producing Clostridium cluster XIVa species specifically colonize mucins in an in vitro gut model. ISME J, 6, 335-340 (2013). 
Vangoitsenhoven, R., Cresci, G.A.M. Role of microbiome and antibiotics in autoimmune diseases. Nutr. Clin. Prac. (in press) (2020) DOI: 10.1002/ncp.10489. Varum, F., Freire, A.C., Bravo, R., Basit, A.W. OPTICORE ${ }^{T M}$, an innovative and accurate colonic targeting technology. Int. J. Pharm. 583, 119372 (2020a).

Varum, F., Freire, A.C., Fadda, H.M., Bravo, R., Basit, A.W. A dual pH and microbiotatriggered coating (Phloral ${ }^{\mathrm{TM}}$ ) for fail-safe colonic drug release. Int. J. Pharm. 583, 119379 (2020b).

Walker, A.W., Sanderson, J.D., Churcher, C., Parkes, G.C., Hudspith, B.N., Rayment, N., Brostoff, J., Parkhill, J., Dougan, G., Petrovska, L. High-throughput clone library analysis of the mucosa-associated microbiota reveals dysbiosis and differences between inflamed and non-inflamed regions of the intestine in inflammatory bowel disease. BMC Microbiology, 11, 7 (2011).

Yadav, V., Varum, F., Bravo, R., Furrer, E., Bojic, D., Basit, A.W. Inflammatory bowel disease: Exploring gut pathophysiology for novel therapeutic targets. Transl. Res. 176, 38-68 (2016).

Yan, P.-G., Li, J.-N. Advances in the understanding of the intestinal microenvironment and inflammatory bowel disease. Chin. Med. J. 133, 834-841 (2020) Zhuang, X., Li, T., Li, M., Huang, S., Qiu, Y., Feng, R., Zhang, S., Chen, M., Xiong, L., Zeng, Z. Systematic review and meta-analysis: Short-chain fatty acid characterization in patients with inflammatory bowel disease. Inflamm. Bowel Dis. 25, 1751-1763 (2019). 


\begin{tabular}{lccc}
\hline Phyla & Donor 1 & Donor 2 & Donor 3 \\
\hline Verrucomicrobia & 0.0 & 3.8 & 0.0 \\
Proteobacteria & 0.2 & 0.7 & 2.2 \\
Firmicutes & 85.9 & 52.9 & 68.1 \\
Bacteroidetes & 13.1 & 6.5 & 19.6 \\
Actinobacteria & 0.8 & 36.0 & 10.2 \\
\hline
\end{tabular}

Table 1: Composition (\%) at the phyla level of the microbiotas of three donor samples from patients with UC before analysis. 


\begin{tabular}{lcccccccccccccc}
\hline Phyla & \multicolumn{1}{c}{ Lumen } & & & \multicolumn{1}{c}{ Mucus } \\
& Donor 1 & Donor 2 & Donor 3 & Donor 1 & Donor 2 & Donor 3 \\
& C & P & C & P & C & P & C & P & C & P & C & P \\
& 35 & 50 & 41 & 39 & 45 & 45 & 48 & 58 & 38 & 48 & 21 & 35 \\
\hline Firmicutes & 23 & 15 & 8 & 5 & 10 & 3 & 1 & 3 & 4 & 9 & 0 & 1 \\
Proteobacteria & 23 & & & & & & & & & & & & \\
Bacteroidetes & 41 & 33 & 23 & 35 & 37 & 32 & 49 & 37 & 34 & 31 & 17 & 7 \\
Verrucomicrobia & 0 & 0 & 26 & 14 & 0 & 0 & 0 & 0 & 11 & 10 & 0 & 0 \\
Actinobacteria & 1 & 2 & 2 & 7 & 8 & 20 & 2 & 2 & 11 & 3 & 61 & 58 \\
\hline
\end{tabular}

Table 2: Composition (\%) of the planktonic (lumen) and mucin-associated (mucus) microbiota at the phyla level of the three UC donors after $48 \mathrm{~h}$ in the M-SHIME ${ }^{\circledR}$ system (C, control; $\mathrm{P}$, dosing with probiotic). 


\begin{tabular}{lll}
\hline Sample & TEER & $\mathrm{n}$ \\
\hline Donor 1 - control & $67 \pm 5$ & 3 \\
Donor 1 - treatment & $80 \pm 8$ & 3 \\
Donor 2 - control & $70 \pm 3$ & 3 \\
Donor 2 - treatment & $82 \pm 7$ & 3 \\
Donor 3 - control & $69 \pm 3$ & 3 \\
Donor 3 - treatment & $83 \pm 5$ & 3 \\
CM (control) & $68 \pm 1$ & 8 \\
\hline
\end{tabular}

Table 3: Effect of colonic batch samples from patients with UC on the transepithelial electrical resistance of the Caco-2/THP1 co-cultures. TEER was measured $24 \mathrm{~h}$ after dosing with probiotic and each value was normalised to its corresponding initial value. 


\begin{tabular}{llll}
\hline Sample & Mean & $\mathrm{n}$ & Significance \\
\hline $\mathrm{CM}$ & $75.2 \pm 2.0$ & 5 & \\
Sodium butyrate & $46.1 \pm 2.0$ & 7 & \\
Donor 1 control & $78.2 \pm 3.9$ & 2 & \\
Donor 1 with Symprove & $64.1 \pm 1.9$ & 3 & $* *$ \\
Donor 2 control & $80.3 \pm 1.1$ & 3 & \\
Donor 2 with Symprove & $59.4 \pm 1.8$ & 3 & $* * *$ \\
Donor 3 control & $80.5 \pm 3.1$ & 3 & \\
Donor 3 with Symprove & $55.5 \pm 2.9$ & 3 & $* * *$ \\
\hline
\end{tabular}

Table 4: Wound area values after $24 \mathrm{~h}$ following dosing with complete medium (CM), sodium butyrate and colonic samples from Donors 1 and 2 with and without dosing with probiotic. Each value is expressed as a percentage and was normalised to its corresponding initial value. $\left({ }^{*}\right)$ represents statistically significant differences for donor samples with and without Symprove; $p<0.01(* *) ; p<0.001\left(^{* * *}\right) ; p<0.0001$ $(* * * *)$ 

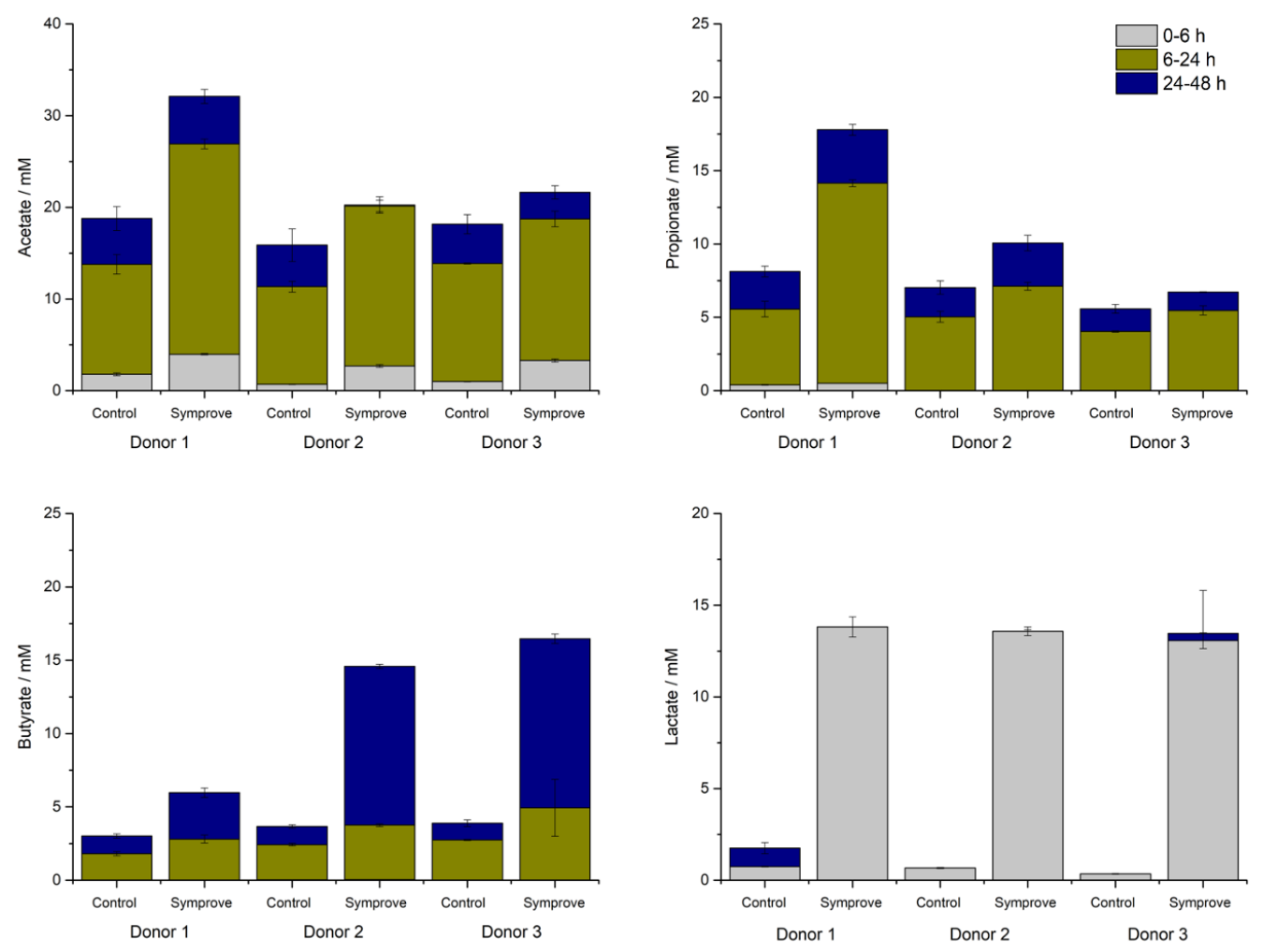

Figure 1. SCFA concentrations in the microbiotas of UC donors as a function of incubation time with probiotic (Symprove). Acetate (top left), propionate (top right), butyrate (bottom left) and lactate (bottom right). Data are shown as mean +/- standard deviation across all donors (3). 


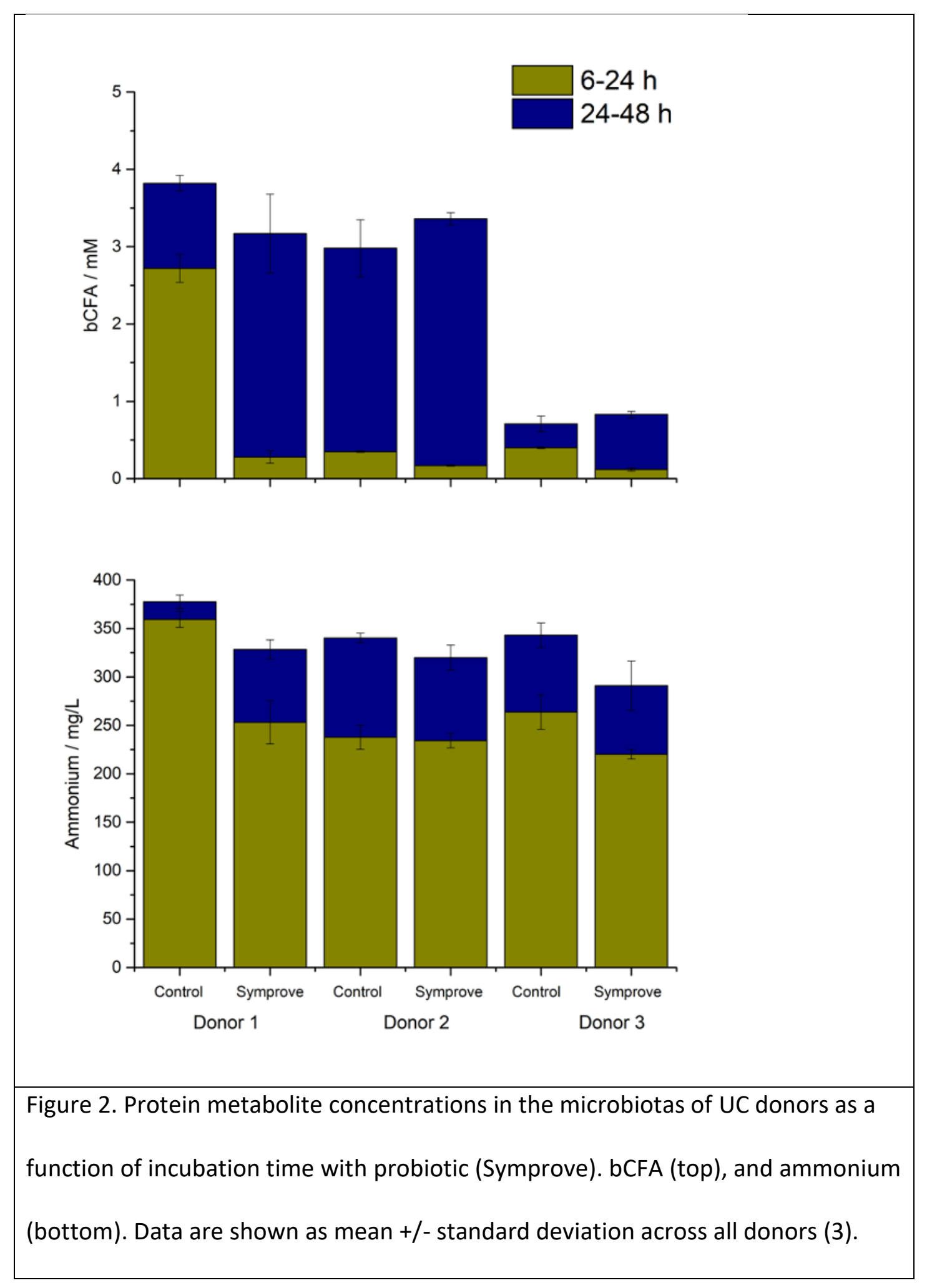









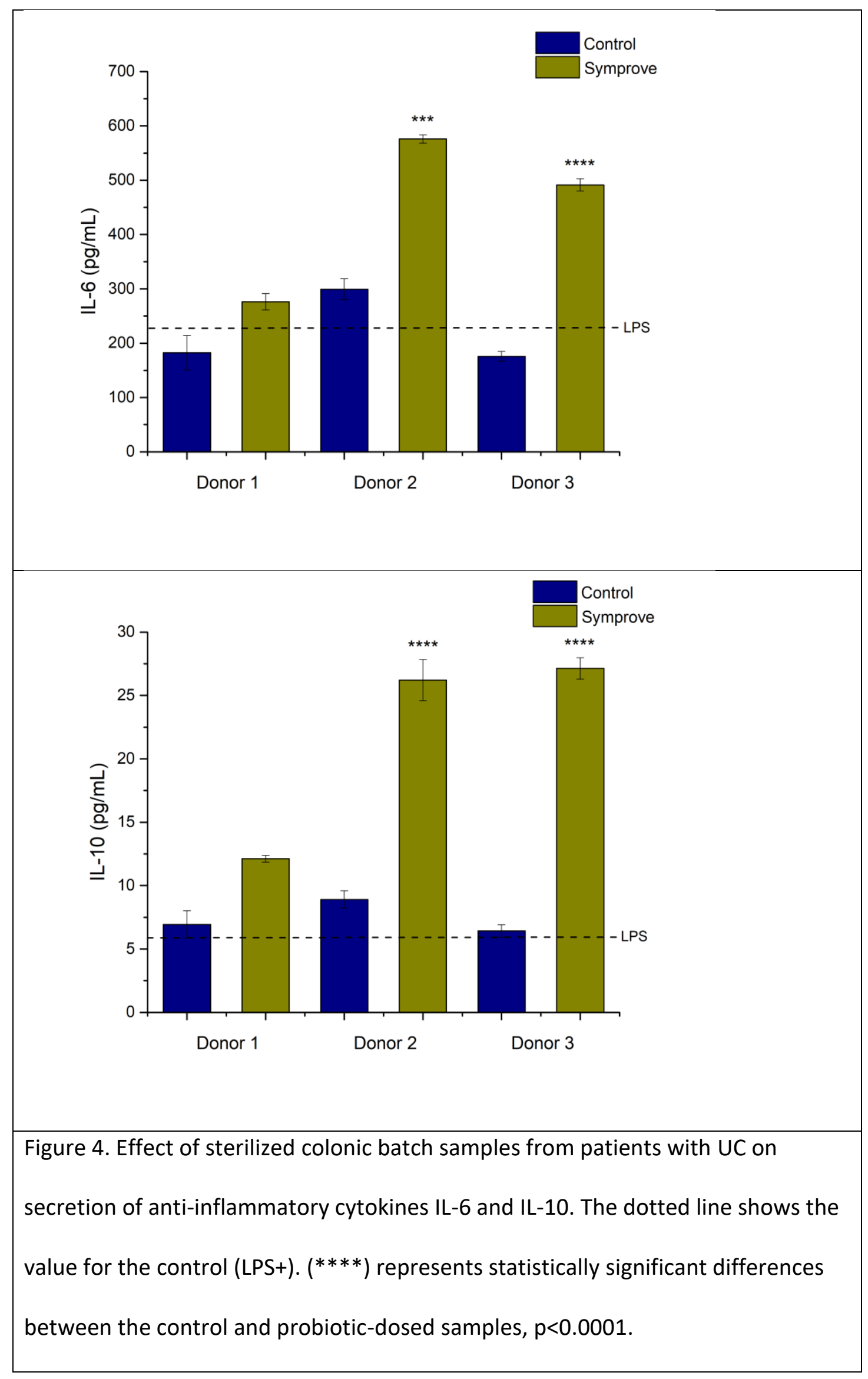





Figure 5. Effect of sterilized colonic batch samples from patients with UC on secretion of inflammatory cytokines TNF $\alpha$, CXCL 10, IL-8 and MCP-1. The dotted line shows the value for the control (LPS+). $\left({ }^{*}\right)$ represents statistically significant differences between the control and probiotic-dosed samples; $p<0.05\left({ }^{*}\right) ; p<0.01$ $(* *)$. 


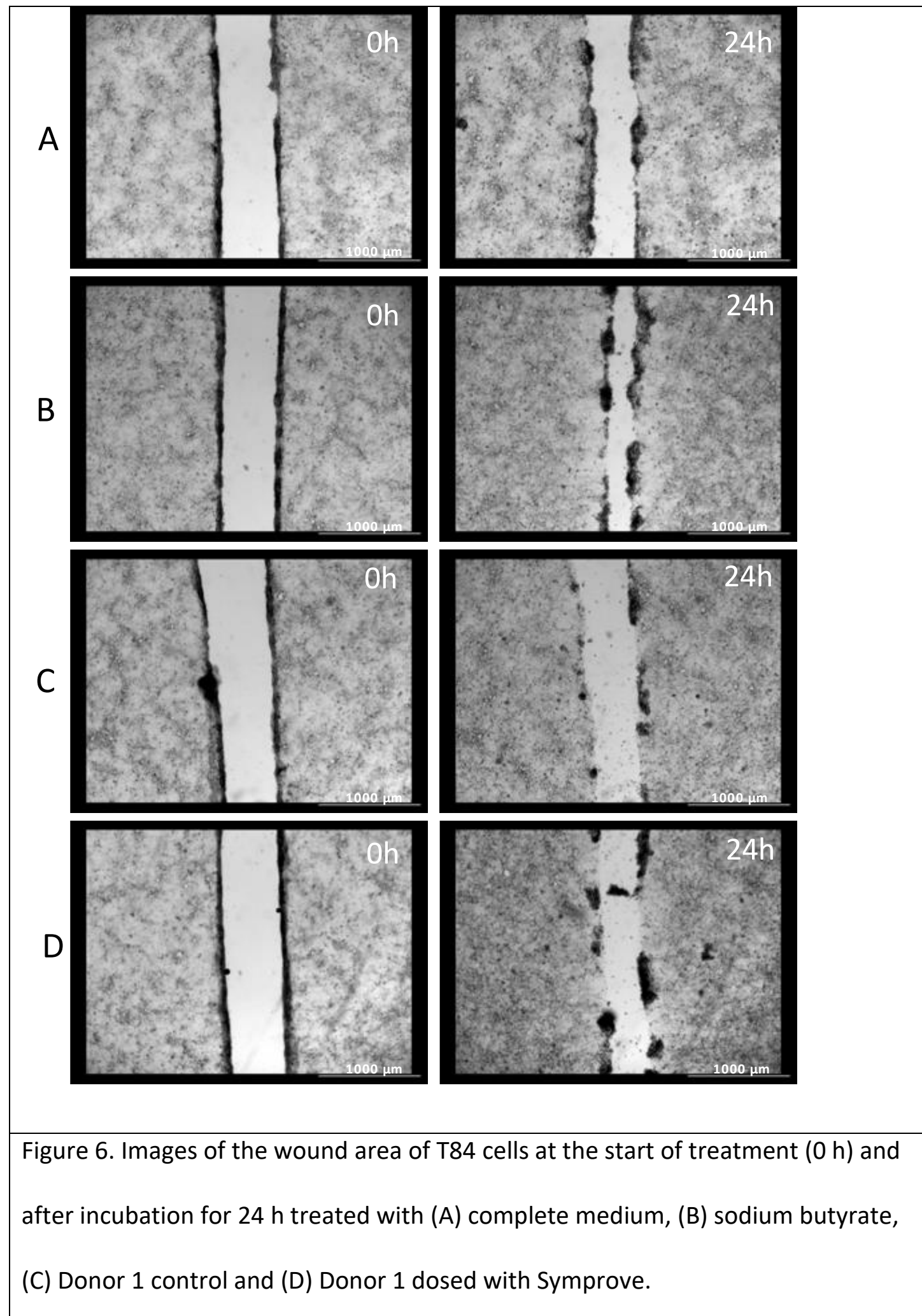

